\title{
Uso de PRFC para mejorar las propiedades mecánicas del concreto: una revisión
}

\section{Use of CFRP to improve the mechanical properties of concrete: a review}

Sócrates Pedro Muñoz Pérez ${ }^{1}$ Diego Mauricio Barboza Chunga ${ }^{1}$, Richard Iván Burga Rafael ${ }^{1}$

1 Universidad Señor de Sipán, Perú

\section{RESUMEN}

El polímero reforzado con fibra de carbono (PRFC) hoy en día se ha vuelto una alternativa de material de fortalecimiento para las estructuras en la construcción porque mejora las propiedades mecánicas del concreto y aunque es un proceso de fabricación de costo elevado, origina un gran beneficio para la estructura de concreto armado (CA). El objetivo de este trabajo ha sido revisar la investigación notable publicada en revistas indexadas de los años 2012 a 2021 (41 artículos de Scopus, 7 de EBSCO, 2 de Science Direct y 1 de Redalyc), sobre el uso del polímero reforzado con fibra de carbono en elementos estructurales, como reforzamiento interno o externo, que beneficia a la estructura en su periodo de vida, al impacto ambiental que este produce, al costo del material y al manejo del material in situ.

PALABRAS CLAVE: PRFC; concreto; propiedades mecánicas; impacto ambiental.

\section{ABSTRACT}

Carbon fiber reinforced polymer (CFRP) today has become an alternative of strengthening material for structures in construction because it improves the mechanical properties of concrete and although it is a high cost manufacturing process, it originates a great benefit for the reinforced concrete structure (CA). The objective of this work has been to review the remarkable research published in indexed journals from the years 2012 to 2021 ( 41 articles from Scopus, 7 from EBSCO, 2 from Science Direct and 1 from Redalyc), on the use of fiber reinforced polymer Carbon in structural elements, such as internal or external reinforcement, which benefits the structure in its lifetime, the environmental impact it produces, the cost of the material and the handling of the material in situ.

KEYWORDS: CFRP; concrete; mechanical properties; environmental impact.

\section{Correspondencia:}

DESTINATARIO: Sócrates Pedro Muñoz Pérez INSTITUCIÓN: Universidad Señor de Sipán DIRECCIÓN: Km 5 Carretera a Pimentel, C. P. 1400, Chiclayo - Perú CORREO ELECTRÓNICO: msocrates@crece.uss.edu.pe
Fecha de recepción: 4 de marzo de 2021. Fecha de aceptación: 18 de junio de 2021. Fecha de publicación: 29 de junio de 2021.
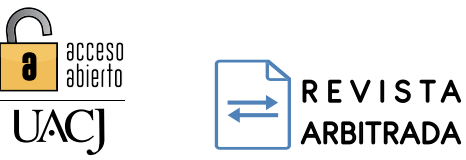

Licencia Creative Commons ARBITRADA

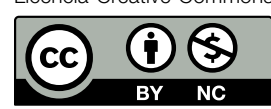




\section{INTRODUCCIÓN}

En las últimas décadas, los polímeros reforzados con fibras (PRF) se han usado ampliamente en aplicaciones civiles, especialmente en el campo del mantenimiento y el refuerzo. Se han llevado a cabo un gran número de investigaciones analíticas y experimentales sobre los elementos de concreto armado (CA), a modo de reforzamientos con varillas, tiras o láminas de $\mathrm{PRF}$, para conocer sus comportamientos mecánicos y se han alcanzado logros fructíferos ${ }^{[1]}$.

Estos polímeros se han utilizado en la ingeniería civil para mejorar la capacidad de carga en los muros de corte, pilares y vigas, entre otros elementos estructurales, pues presentan una adecuada relación resistencia-peso, resistencia a la tensión y durabilidad [2], e incluso alta resistencia a la corrosión, por lo cual los materiales PRF se utilizan a menudo para el refuerzo en elementos estructurales de concreto armado que se encuentran expuestos a la humedad [3].

Una parte a considerar en las edificaciones de concreto armado existentes son los diseños con códigos estructurales antiguos, siendo estos vulnerables a eventos extremos, como los terremotos, y por ello se debe considerar la integración de polímeros reforzados con fibra. Debido a sus excelentes propiedades mecánicas, facilidad de aplicación y eficacia, han sido ampliamente contemplados para el reforzamiento en diferentes tipos de edificaciones porque tienen un comportamiento apropiado en las estructuras [2].

Existen diversos tipos de fibras que se pueden agregar al concreto, entre ellos el basalto, el vidrio, la aramida y el carbono. El polímero reforzado con fibra de carbono (PRFC) se caracteriza por un alto módulo de Young equivalente al del acero. Esta propiedad de rigidez favorable hace que el refuerzo de PRFC sea la opción preferida para mantener las deflexiones en las estructuras de los edificios al mínimo, como se requiere en el estado límite de servicio ${ }^{[4]}$.

El peso unitario del PRFC oscila entre 1.5 y $1.7 \mathrm{~g} / \mathrm{cm}^{3}$, que es cinco veces más ligero que el del acero convencional y la resistencia a la tensión del PRFC es de 8 a 10 veces mayor que la de este material. Las fibras de PRFC tienen un módulo de elasticidad de $165 \mathrm{GPa}$ y su relación de Poisson es 0.183. Numerosos estudios han descubierto que el concreto reforzado con fibras de carbono tiene una mayor resistencia a la tensión, una mayor fuerza a las fisuras y una mejor tenacidad en comparación con el concreto con polímeros de otras fibras ${ }^{[5]}$.

Por lo tanto, el uso de polímero reforzado con fibra de carbono posee ventajas como alta resistencia y rigidez, así como plasticidad y durabilidad. Además de las cargas regulares, los fabricantes modernos de PRFC también se preocupan por los fuertes impactos ambientales $\mathrm{y}$ accidentales sobre las estructuras de concreto, como terremotos, tornados y explosiones [5].

Las estructuras compuestas reforzadas con fibras se han convertido en materiales de ingeniería muy competitivos en los últimos años y han ido sustituyendo gradualmente a los materiales metálicos convencionales y a otros materiales poliméricos en muchas industrias importantes [6].

\section{METODOLOGÍA}

En la presente revisión se examinaron bases de datos y en ellas se encontraron 1os artículos indexados siguientes: 41 en Scopus, 2 en Science Direct, 7 en EBSCO y 1 en Redalyc, de los cuales 14 son de 2021, 28 de 2020, 5 de 2019, 1 de 2018, 1 de 2015, 1 de 2013 y 1 de 2012. Las palabras clave que se han empleado para la búsqueda han sido polímeros reforzados con fibras de carbono, resina epoxi, polímero reforzado con fibra y sostenibilidad estructural. A continuación, para un mejor detalle, en la Tabla 1 se presentan los artículos seleccionados, según el año de publicación con respecto a su base de datos.

TABLA 1

Artículos Revisados, Por Base de Datos

\begin{tabular}{|l|c|c|c|c|c|c|c|c|}
\hline \multirow{2}{*}{$\begin{array}{c}\text { BASE DE } \\
\text { DAtOS }\end{array}$} & \multicolumn{7}{|c|}{ AÑo } & \multirow{2}{*}{ TotAL } \\
\cline { 2 - 9 } & 2012 & 2013 & 2015 & 2018 & 2019 & 2020 & 2021 & \\
\hline Scopus & & 1 & & 1 & 5 & 21 & 13 & 41 \\
\hline $\begin{array}{l}\text { Science } \\
\text { Direct }\end{array}$ & 1 & & & & & 1 & & 2 \\
\hline EBSCO & & & & & & 6 & 1 & 7 \\
\hline Redalyc & & & 1 & & & & & 1 \\
\hline Total & 1 & 1 & 1 & 1 & 5 & 28 & 14 & 51 \\
\hline
\end{tabular}

\section{RESULTADOS Y DISCUSIÓN}

\section{A. Origen del polímero reforzado con fibra}

La aplicación de elementos compuestos con polímero reforzado con fibra comenzó a finales de la Segun- 
da Guerra Mundial en aplicaciones como la aviación, y a finales de los años 80 su uso llegó al sector de la construcción y las infraestructuras. El primer proyecto de investigación sobre la opción de utilizar materiales compuestos de PRF para reforzar estructuras de concreto se remonta al año 1978 en Alemania. Sin embargo, las primeras aplicaciones prácticas tuvieron lugar en Suiza, con el fin de aumentar la capacidad portante a flexión de los puentes de concreto armado [7].

A partir del año 1982 se dieron los primeros estudios en el comportamiento de la columna de concreto confinado con PRF bajo flexión uniaxial [8].

El polímero reforzado con fibra, que se utiliza habitualmente para la rehabilitación, puede aplicarse en el concreto armado tanto en el lado de tensión como en el lado de esfuerzo, con el fin de resistir la corrosión de las barras de acero. En [9], los investigadores abordaron el tema del control de la corrosión de las estructuras de CA y llegaron a la conclusión de que el encamisado favorece la disminución en la tasa de corrosión de estas estructuras.

El sistema de anclaje juega un papel vital en las estructuras reforzadas con PRF y se han desarrollado varios tipos de sistemas de anclaje, por lo cual el refuerzo en una placa de concreto armado es más eficaz debido a su mayor grosor, sin embargo, esto origina que sea difícil de fijarse en la placa de concreto armado [10].

\section{B. Origen de las fibras de carbono}

La utilización de polímeros reforzados con fibra de carbono es una tecnología de refuerzo para estructuras de concreto que se ha adoptado en proyectos de rehabilitación y de reforzamiento durante los últimos 25 años [11].

La primera vez que se añadió fibras de carbono de un determinado tamaño y especificación en el concreto, el material se comportó en función de autodetección del esfuerzo interno, la deformación y los daños [12].

Los materiales compuestos de fibra de carbono son materiales ligeros que son adecuados para reducir el peso y tienen gran influencia en las propiedades mecánicas (módulo de Young y tasa de liberación de energía de deformación), por lo tanto, cumple con los requisitos de rendimiento mecánico [13].
Las fibras de carbono se obtienen del tejido de carbono, método que consiste en el entrelazado de filamentos de este material ${ }^{[14]}$.

La forma más empleada para producir fibra de carbono es la siguiente: como primer paso se usa un horno industrial con una temperatura de $300{ }^{\circ} \mathrm{C}$, estirando la materia y originando una formación de las partículas para estabilizar; luego el horno se eleva a una temperatura de $1000{ }^{\circ} \mathrm{C}$, calcinando elementos como el hidrógeno o el nitrógeno y presenta moléculas de carbono orientándose de manera hexagonal a lo extenso de la fibra; y, por último, el horno se eleva a una temperatura de $2000{ }^{\circ} \mathrm{C}$, con la finalidad de aplicar un catalizador que proporciona adherencia a las fibras. El compuesto final son filamentos de carbono con una pureza que van desde del 95\% hasta el 99\%. Este componente es trefilado a diámetros desde 5-10 $\mu \mathrm{m}$, siendo cinco veces más delgado que el cabello humano [15]. Por ello, las propiedades de las fibras de carbono no cambian en una atmósfera no oxidante a $2000^{\circ} \mathrm{C}$, a diferencia de la fibra vidrio o fibra de aramida ${ }^{[16]}$.

Las especificaciones técnicas de las fibras de carbono dependen del tipo de precursor y del procedimiento de producción, que son el poliacrilonitrilo (PAN) y la carbonización de la fibra de carbono. Se utilizan en diferentes formas de filamentos largos o cortos, picados, molidos, tejidos y esteras no tejidas, para la fabricación de PRFC. Ha dado lugar a muchas aplicaciones para sustituir los materiales como el acero, el aluminio y las aleaciones en diferentes industrias [17].

Los laminados de materiales con fibras de carbono (FC) se encuentran formados por dos componentes destacados: la resina epoxi, que corresponde a la matriz, y los carbonos, que son las fibras. Este compuesto proporciona un apropiado comportamiento hasta en tres direcciones, todo ello en relación a la designación de las fibras [18].

\section{Resina epoxi}

Las resinas epoxi (RE) son matrices termoendurecibles [19]. En este caso, el material de la matriz del refuerzo de PRFC está compuesto en su totalidad por resina epoxi [4].

Las siguientes propiedades hacen que las resinas epóxicas sean especialmente adecuadas para su uso como sistema de matriz: excelente comportamiento mecánico 
y térmico, buena adherencia con la fibra de refuerzo, aceleración del proceso de curado a diferentes temperaturas y baja contracción después de alcanzar el punto de gelificación [6].

Por lo tanto, la resina epóxica se presenta como la matriz de los PRFC porque es una de las matrices de PRF más utilizadas en la ingeniería civil. El PRFC presenta resistencias a la tensión mayores a $350 \mathrm{~kg} / \mathrm{cm}^{2}$ con el concreto ${ }^{[20]}$.

Sin embargo, debido a su propiedad termoestable, los materiales basados en resinas termoestables se destruyen fácilmente por factores mecánicos, químicos, térmicos y de radiación UV, lo que inevitablemente conduce a daños locales y a la generación de microgrietas en el material difíciles de detectar a tiempo [21].

La inserción de una capa intermedia discreta de termoplástico entre las capas adyacentes es una de las estrategias más populares para mejorar la resistencia a la fractura interlaminar del laminado de la fibra de carbono y la resina epoxi (FC/RE). Se han utilizado muchas sustancias para reforzar los laminados, incluidas los nanomateriales de carbono, los termoplásticos y otras. Los materiales para la capa intermedia más frecuentes son los termoplásticos en forma de polvo, película, malla y tela no tejida, que se utilizan para el endurecimiento de esta capa [22].

\section{Uso de PRFC}

El uso de PRFC se puede dar de dos formas: de refuerzo interno y refuerzo externo.

Los laminados de PRFC en el refuerzo externo se están convirtiendo en los materiales preferidos en las aplicaciones de refuerzo en el concreto armado debido a sus ventajas superiores sobre el revestimiento de acero convencional [23].

La interacción con la superficie del concreto se encuentra compuesto por adhesión, fricción y unión mecánica, y la unión interna se refiere a la transferencia de esfuerzos cortantes desde el interior del elemento estructural, presentando una mayor resistencia [24].

Los tratamientos internos de las varillas de PRFC que se aplican en un refuerzo interno en el CA, muestran una resistencia mecánica fiable, evitando el deslizamiento interfacial entre el PRFC y el adhesivo epóxico [25].

El encamisado de PRFC, tanto para un refuerzo interno o un refuerzo externo, se utiliza con mayor frecuencia para aumentar las capacidades sísmicas de los pilares y otros elementos estructurales, sin embargo, puede no ser factible debido a problemas de accesibilidad y dar lugar a dimensiones excesivas no deseadas del elemento reforzado ${ }^{[26]}$.

La fibra de carbono no pierde eficacia en sus propiedades ante un ambiente húmedo y caliente, sin embargo, las propiedades de los adhesivos epoxídicos utilizados en los materiales PRF se degradan significativamente en un entorno húmedo y caliente. La resistencia de la unión de los adhesivos disminuye rápidamente cuando se acerca a temperaturas con transiciones vítreas [27].

El proceso del fallo a compresión uniaxial del PRFC se divide en cuatro fases: elástica lineal, de desarrollo estable de grietas, de desarrollo inestable de grietas y descendente, de las cuales las grietas principales cambian de grado de resistencia del concreto y la dosificación de las FC [28].

A continuación, para un mejor detalle, en la Tabla 2 se presenta las propiedades generales de las fibras de acero (FA), fibra de vidrio (FV) y fibra de carbono (FC).

TABLA 2

Propiedades Generales de las Fibras de Acero, de Vidrio y DE CARBono

\begin{tabular}{|l|c|c|}
\hline \multicolumn{1}{|c|}{ Fibra } & $\begin{array}{c}\text { RESISTENCIA A LA } \\
\text { TENSIÓN (MNPA) }\end{array}$ & $\begin{array}{c}\text { Módulo DE } \\
\text { Elasticidad (GPA) }\end{array}$ \\
\hline Acero & 1700 & 210 \\
\hline Vidrio & 2500 & 72 \\
\hline Carbono & 3200 & 380 \\
\hline
\end{tabular}

\section{E. Ventajas del uso de PRFC}

Los PRFC se han utilizado ampliamente en estructuras de ingeniería civil, debido al desgaste de las barras de acero por la corrosión, lo que reduce en gran medida el ciclo de vida de las estructuras de concreto armado. Las ventajas del uso de PRFC para el concreto armado son su resistencia a la corrosión, su peso ligero, su alta resistencia a la tensión y buena durabilidad. Ha atraído una gran atención en el campo de la ingeniería de nuevas construcciones, así como en la rehabilitación de estructuras de ingeniería [29]. 


\section{F. Aplicación de PRFC en estructuras}

\section{Puentes con reforzamiento PRFC}

Debido al complejo entorno de la industria del transporte actual, la sobrecarga de grandes camiones se ha convertido en un fenómeno común. Los movimientos de estos grandes vehículos debilitan la seguridad del puente. Por lo tanto, es necesario analizar la respuesta dinámica acoplada vehículo-puente antes y después del refuerzo con PRFC [30].

Para los puentes de concreto armado o pretensado, existen métodos de refuerzo establecidos, como el pretensado externo adicional, la inserción de conectores a cortante, concreto adicional en la zona de compresión o aplicación externa de aplicación externa de PRFC en forma de láminas o laminillas. Ello se interpreta en una utilización más eficaz de los requerimientos, presentando un menor peso muerto adicional y una mayor vida útil [31].

Las láminas con polímero reforzado con fibra de carbono se aplican a fin de refuerzo externo en los componentes débiles, especialmente las vigas de concreto armado que corren un alto riesgo de degradación rápida durante el funcionamiento del puente, debido a los impactos del peso de la superestructura y las cargas del tráfico. Las tensiones normales desarrolladas en una capa de PRFC adherida pueden causar delaminación, como se indica en la norma ACI 440.2R-02 [32].

La aplicación de los cables de PRFC en puentes atirantados se limita principalmente a los puentes de corto alcance y no a los de largo alcance de gran envergadura, debido principalmente al elevado costo de los cables de PRFC, que restringe su aplicación en puentes de gran luz, y a que la rigidez de estos puentes es relativamente baja. Por tanto, la instalación de los cables de PRFC es un reto al aplicarla en el uso constructivo [33].

\section{Pilotes con PRFC}

El pilote de concreto armado es una estructura compuesta. El concreto tiene una excelente resistencia a la compresión, pero poca resistencia a la tensión. La resistencia y ductilidad de los pilotes pueden disminuir rápidamente debido a la corrosión del material en ambientes cálidos y húmedos. Los compuestos de PRFC se consideran un nuevo material para superar la debilidad de los pilotes [34].
El refuerzo que se puede dar a los pilotes es tanto de reforzamiento externo como interno, ofreciendo al elemento estructural un control de reparación o control preventivo. En la Figura 1 se aprecia el reforzamiento externo de PRFC en un pilote rectangular de concreto.

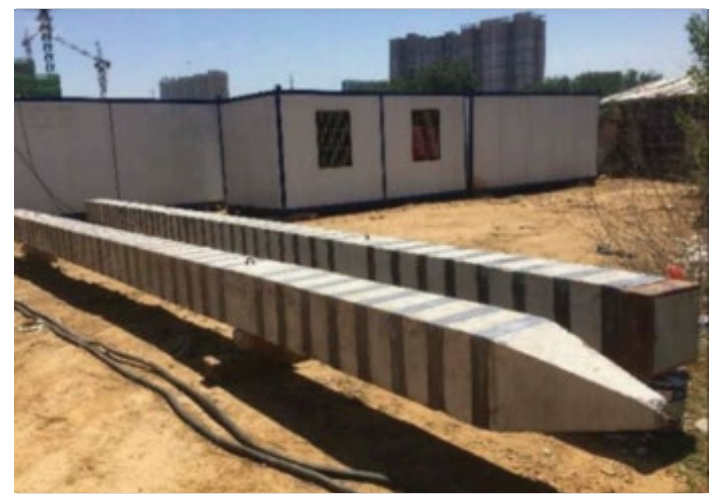

Figura 1. Pilote rectangular de concreto con aplicación de PRFC. Fuente: [35].

\section{Columnas con PRFC}

El polímero reforzado con fibra de carbono, como refuerzo interno o externo, comúnmente se utiliza para envolver columnas de concreto con el fin de mejorar la capacidad de carga de compresión axial. Emplear tiras o encamisados de laminados de PRFC puede ser un método eficaz de rehabilitación y refuerzo estructural [36]. En la Figura 2 se aprecia el reforzamiento enrollado de PRFC en una columna de concreto armado.

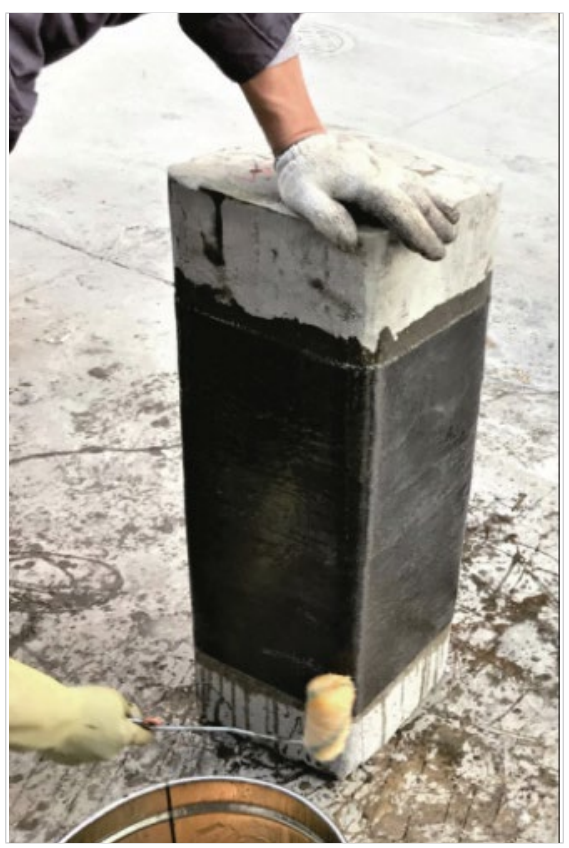

Figura 2. Proceso de reforzamiento de columna con enrollado de PRFC. Fuente: [37]. 
Vigas con PRFC

El rendimiento de las vigas de concreto armado, en términos de flexión, cortante o ambos, puede mejorar significativamente mediante la fijación de compuestos de polímero reforzado con fibra de carbono en las superficies exteriores para la viga. A pesar de utilizar un adhesivo especial de acuerdo con las recomendaciones del fabricante, es frecuente encontrar problemas de despegue, especialmente en los extremos de los PRFC o cerca de las regiones con grandes tensiones o agrietadas [38].

Las varillas de PRFC en vigas son colocadas de forma vertical u horizontal y presentan un aumento a la resistencia al corte en el rango de $17 \%$ a $25 \%$ [39]. En la Figura 3 se aprecia el reforzamiento externo en una viga con laminado de PRFC de concreto armado.

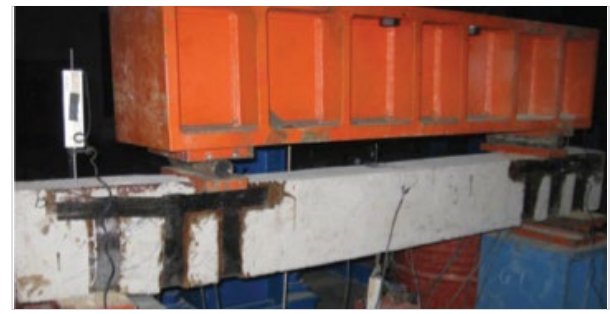

Figura 3. Reforzamiento externo a vigas con laminados de PRFC. Fuente: ${ }^{[40] .}$

\section{Losas con PRFC}

Trabajos recientes han identificado los paneles de barras de PRF de carbono como un refuerzo unido externamente y una alternativa a los laminados empalmados. Las varillas de PRFC de pequeño diámetro se colocan lado a lado a una distancia discreta para formar un panel. Los paneles vecinos se juntan y se hacen continuos mediante un mecanismo de finger joint [41]. En la Figura 4 se aprecia el reforzamiento externo de laminados de PRFC en una losa de concreto armado.

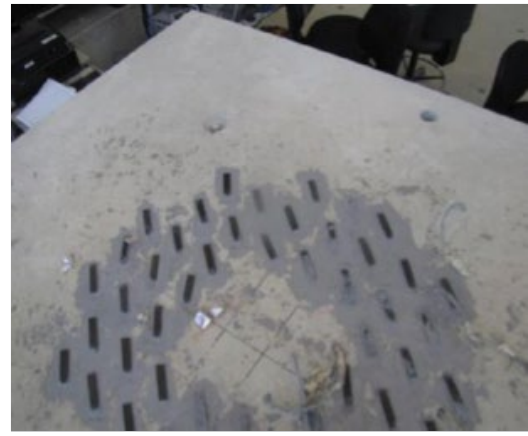

Figura 4. Losa con reforzamiento de laminados con PRFC. Fuente: ${ }^{[42]}$.
El uso de tiras de PRFC con ranuras es muy útil para aumentar la resistencia, la capacidad de servicio y los factores de rendimiento, de rigidez y de tenacidad en la viga de concreto armado en el manejo de tiras con PRFC como refuerzo externo sin ranuras [38].

El pretensado de las varillas de PRFC es una forma eficaz de mejorar el comportamiento estructural de las vigas reforzadas con fibras de carbono de pared delgada. Esto es especialmente evidente en la capacidad de carga de la cortante ${ }^{[43]}$.

El comportamiento de adhesión del PRFC pretensado al concreto conlleva grandes deformaciones, por ende, es importante considerar un mayor avance en la tecnología de fibra de carbono en las estructuras debido a que existen anomalías [44].

También se recomienda que la resina utilizada para los PRFC se acerque más a las propiedades del epoxi, debido a que presenta propiedades más fiables [45].

Además, las fibras de carbono en un ambiente seco a lo largo del concreto muestran una mejor resistencia a la compresión y al fallo por deformación, así como a una mejor conductividad [46].

Por otra parte, las láminas de PRFC tienen un efecto significativo en el comportamiento cíclico de las vigas de CA, mejorando así la resistencia máxima y el desplazamiento último hasta aproximadamente el $66.67 \%$ y el $77.14 \%$ en vigas ${ }^{[47]}$.

El refuerzo con confinamiento de PRFC es una forma útil de mejorar el comportamiento sísmico de los pilares mal reparados, en términos de resistencia, capacidad de disipación de energía y ductilidad [48].

Asimismo, el PRFC es una alternativa muy prometedora y efectiva para prevenir el fallo abrupto de las columnas con encamisado de baja carga axial concéntrica. Con la aplicación del encamisado de PRFC no uniforme en el refuerzo externo, las columnas de concreto armado mejoran sustancialmente la resistencia, la ductilidad y la absorción de energía [49].

Respecto al impacto ambiental, el sector de los materiales de construcción es el tercer sector industrial que más dióxido de carbono emite en todo el mundo. Se estima que la producción de cemento representa el 7\% del total 
de las emisiones antropogénicas de $\mathrm{CO}_{2}$, por lo cual la incorporación de PRFC en estructuras de CA favorece la sostenibilidad y durabilidad en el ciclo de vida de las edificaciones y es, por tanto, un factor que genera una buena gestión de los recursos naturales, reduciendo los niveles actuales de presión antropogénica sobre el ambiente ${ }^{[50]}$.

En el aspecto de la economía, el costo del refuerzo de laminado de PRFC en el año 2020 en la ciudad Hong Kong fue de aproximadamente de 39.68 dólares estadounidenses (USD), mientras que las barras de refuerzo de acero convencional su costo fue de 47.46 USD. Esto implica que el refuerzo de PRFC es un $16.4 \%$ más barato que los de acero, según el escenario considerado [51].

\section{CONCLUSIONES}

El uso de polímeros reforzados con fibra de carbono en el concreto armado es recomendable porque brinda seguridad, con el propósito de que las estructuras puedan superar diferentes tipos de demandas de esfuerzo excepcionales que puedan provocarse, ofreciendo características excelentes para la asimilación de fuerzas y escasa vulnerabilidad.

Actualmente, el uso de la fibra de carbono en las construcciones es costoso en comparación con otros materiales, pero los beneficios lo hacen competitivo.

Una de las características resaltantes del refuerzo de PRFC es que presenta resistencia a la corrosión, lo que conlleva a que la estructura alargue su ciclo de vida y redunde en menores gastos de mantenimiento en la construcción.

La fibra de carbono es un material que se puede reciclar, lo que lo coloca como un producto de impacto ambiental positivo.

Por ello, la utilización de PRFC en el concreto armado garantiza un rendimiento óptimo de la estructura.

\section{REFERENCIAS}

[1] N. Al-Akhras y M. Al-Mashraqi, "Repair of corroded self-compacted reinforced concrete columns loaded eccentrically using carbon fiber reinforced polymer", Case Stud. Constr. Mater., vol. 14, no. 476, pp. 1-19, 2021, doi: 10.1016/j.cscm.2020.e00476.
[2] J. Yan, Y. Liu, Z. Xu, Z. Li y F. Huang, "Experimental and numerical analysis of CFRP strengthened RC columns subjected to close-in blast loading", Int J Impact Eng, vol. 146, no. 103720, pp. 1-21, 2020, doi: 10.1016/j. ijimpeng.2020.103720.

[3] L. Jin, H. Chen, Z. Wang y X. Du, "Size effect on axial compressive failure of CFRP-wrapped square concrete: Tests and simulations", Compos. Struct., vol. 254, no. 112843, pp. 1-19, 2020, doi: 10.1016/j. compstruct.2020.112843.

[4] N. Stoiber, M. Hammerl y B. Kromoser, "Cradle-togate life cycle assessment of CFRP reinforcement for concrete structures: Calculation basis and exemplary application", J. Clean. Prod., vol. 280, no. 124300, pp. 1-12, 2021, doi: 10.1016/j.jclepro.2020.124300.

[5] G.-J. Liu, E.-L. Bai, J.-Y. Xu, N. Yang y T.-j. Wang, "Dynamic compressive mechanical properties of carbon fiber-reinforced polymer concrete with different polymer-cement ratios at high strain rates", Constr Build Mater., vol. 261, no. 119995, pp. 1-11, 2020, doi: 10.1016/j. conbuildmat.2020.119995.

[6] L. Knapčíková y A. Behúnová, "Research of Casting Moulding of Epoxy Resin Composites Reinforced with High-Strength Fibres during the Manufacturing Operations", TEM Journal, vol. 9, no. 4, pp. 1488-1494, 2020, doi: 10.18421/TEM94-22.

[7] D. Brizuela-Valenzuela, M. N. González-García y A. Cobo-Escamilla, "Influence of the Modulus of Elasticity of CFRPs on the Compressive Behavior of Confined Test Pieces and on the Flexural Behavior of Short Concrete Beams", Appl. Sci., vol. 11, no. 491, pp. 1-17, 2021, doi: 10.3390/app11020491.

[8] R. Gopal, S. Krishnachandran y B. H. Bharatkumar, "Monotonic and cyclic response of hybrid fibre reinforced polymer reinforcing system for reinforced concrete columns under eccentric loading", $A d v$. Struct. Eng., vol. 23, no. 16, pp. 3456-3469, 2020, doi: $10.1177 / 1369433220939209$.

[9] I. K. Dhindsa, H. S. Rai y H. Singh, "Development of rectangular hybrid-stiffened-plate structural system with fibre-reinforced polymer fabric composite in tension zone", Adv. Struct. Eng., vol. 24, no. 2, pp. 211-226, 2021, doi: $10.1177 / 1369433220948755$. 
[10] G. Wang, C. Yang, C. Meng, Z. Xia, Y. Pan y M. Wang, "Experimental study on the mechanical and self-sensing behaviors of prestressed carbon fiber-reinforced polymer reinforced concrete composite structures", $A d v$. Struct. Eng., vol. 23, no. 8, pp. 1507-1520, 2020, doi: $10.1177 / 1369433219895915$.

[11] W. Zhu, M. Li, H. Qin, F. Fu y F. Liu, "Behavior of RC Beams Strengthened Using Steel-Wire-Carbon-Fiber-Reinforced Plates", Materials, vol. 13, no. 3996, pp. 1-16, 2020, doi: 10.3390/ma13183996.

[12] M. A. L. Silva, K. V. Dedigamuwa y J. C. P. H. Gamage, "Performance of severely damaged reinforced concrete flat slab-column connections strengthened with Carbon Fiber Reinforced Polymer", Compos. Struct., vol. 255, no. 112963, pp. 1-10, 2021, doi: 10.1016/j. compstruct.2020.112963.

[13] Y. Zhang, C. Yan, X. Huang y Y. Chen, "Structural Design and Mechanical Performance Analysis of Carbon Fiber Closed Fixtures for UHV Transmission Lines", Math. Probl. Eng., vol. 2021, id. 6105360, pp. 1-13, 2021, doi: 10.1155/2021/6105360.

[14] R. Auriga et al., "Performance properties of plywood composites reinforced with carbon fibers", Compos. Struct., vol. 248, no. 112533, pp. 1-7, 2020, doi: 10.1016/j. compstruct.2020.112533.

[15] M. Moncayo-Theurer, J. Rodriguez, Alcívar, López, Soriano y Villacis, "Las fibras de carbono como una alternativa para reforzamiento de estructuras", Ingeniería, vol. 20, no. 1, pp. 57-62, 2016. [En línea]. Disponible: https://www.redalyc.org/pdf/467/46750927006.pdf

[16] K. Ostrowski, R. Kinasz y P. Dybel, "The impact of surface preparation for self-compacting, high-performance, fiber-reinforced concrete confined with CFRP using a cement matrix", Materials, vol. 13, no. 2830, pp. 1-18, 2020, doi: 10.3390/ma13122830.

[17] E. Pakdel, S. Kashi, R. Varley y X. Wang, "Recent progress in recycling carbon fibre reinforced composites and dry carbon fibre wastes", Resour Conserv Recycl, vol. 166, no. 105340, pp. 1-20, 2021, doi: 10.1016/j. resconrec.2020.105340.

[18] M. Molina, J. J. Cruz, S. Oller, A. H. Barbat y L. Gil, "Estudio numérico-experimental de la interfaz hormi-
gón-epoxi-FRP para una estructura reforzada sometida a doble corte", Rev. int. métodos numér. cálc. diseño ing., vol. 28, no. 2, pp. 65-79, 2012. [En línea]. Disponible: https://www.scipedia.com/public/Molina_et_ al_2012a

[19] G. Mihu, C. V. Ungureanu, V. Bria, M. Bunea y R. Chihai, "The Mechanical Properties of Organic Modified Epoxy Resin", The Annals Of "Dunarea de Jos", University of Galati Fascicle IX. Metallurgy and Materials Science, vol. 38, no. 3, pp. 10-14, 2020, doi: 10.35219/ mms.2020.3.02.

[20] S. Dong, C. Li y G. Xian, "Environmental impacts of glass-and carbon-fiber-reinforced polymer bar-reinforced seawater and sea sand concrete beams used in marine environments: An LCA case study", Polymers, vol. 13, no. 154, pp. 1-16, 2021, doi: 10.3390/ polym 13010154 .

[21] F. Zhang, L. Zhang, M. Yaseen y K. Huang, "A review on the self-healing ability of epoxy polymers", J. Appl. Polym. Sci., vol. 138, no. 50260, pp. 1-14, 2020, doi: 10.1002/app.50260.

[22] D. Quan, R. Alderliesten, C. Dransfeld, N. Murphy, A. Ivanković y R. Benedictus, "Enhancing the fracture toughness of carbon fibre/epoxy composites by interleaving hybrid meltable/non-meltable thermoplastic veils", Compos. Struct., vol. 252, no. 112699, pp. 1-9, 2020, doi: 10.1016/j.compstruct.2020.112699.

[23] K. Helal, S. Yehia, R. Hawileh y J. Abdalla, "Performance of preloaded CFRP-strengthened fiber reinforced concrete beams", Compos. Struct., vol. 244, no. 112262, pp. 1-12, 2020, doi: 10.1016/j.compstruct.2020.112262.

[24] F. Suppanz y B. Kromoser, "Verbundverhalten subtraktiv bearbeiteter CFK-Stäbe in UHPC", Beton- und Stahlbetonbau, vol. 115, no. 8, pp. 1-10, 2020, doi: 10.1002/ best. 201900088 .

[25] X. Wang y L. Cheng, "Bond characteristics and modeling of near-surface mounted CFRP in concrete", Compos. Struct., vol. 255, no. 113011, pp. 1-13, 2021, doi: 10.1016/j.compstruct.2020.113011.

[26] R. Z. Al-Rousan, M. A. Alhassan y R. J. Al-omary, "Response of interior beam-column connections integrated with various schemes of CFRP composites", Case Stud. 
Constr. Mater., vol. 14, no. 488, pp. 1-18, 2021, doi: 10.1016/j.cscm.2021.e00488.

[27] Z. Chen, P. Huang, G. Yao, X. Guo, Y. Yang, W. Li y B. Wu, "Experimental study on fatigue performance of RC beams strengthened with CFRP under variable amplitude overload and hot-wet environment", Compos. Struct., vol. 244, no. 112308, pp. 1-11, 2020, doi: 10.1016/j.compstruct.2020.112308.

[28] B. Liu, J. Zhou, X. Wen , X. Hu y Z. Deng, "Mechanical properties and constitutive model of carbon fiber reinforced coral concrete under uniaxial compression", Constr Build Mater., vol. 263, no. 120649, pp. 1-13, 2020, doi: 10.1016/j.conbuildmat.2020.120649.

[29] Q. Cao, X. Lv, C. Zhou y S. Song, "High strength expansive concrete-encased-steel filled carbon fiber reinforced polymer tubes under axial monotonic and cyclic load", J Compos Mater, vol. 54, no. 29, pp. 4557-4573, 2020, doi: $10.1177 / 0021998320936743$.

[30] X. Cheng, X. Cai, B. Liu y W. Zhang, "Vehicle-bridge coupling dynamic response of a box bridge after reinforcement with prestressed CFRP”, J. Vibroengineering, vol. 22, no. 7, pp. 1715-1730, 2020, doi: 10.21595/ jve.2020.21274.

[31] V. Adam, J. Bielak, C. Dommes, N. Will y J. Hegger, "Flexural and Shear Tests on Reinforced Concrete Bridge Deck Slab Segments with a Textile-Reinforced Concrete Strengthening Layer", Materials, vol. 13, no. 18, pp. 1-25, 2020, doi: 10.3390/ma13184210.

[32] V. H. Mac, J. Huh, N. S. Doan, G. Shin y B. Y. Lee, “Thermography-Based Deterioration Detection in Concrete Bridge Girders Strengthened with Carbon Fiber-Reinforced Polymer", Sensors, vol. 20, no. 3263, pp. 1-19, 2020, doi: 10.3390/s20113263.

[33] S. Sun, K. Mei, Y. Sun, B. Li y H. Huang, "Structural Performance of Super-Long-Span Cable-Stayed Bridges with Steel and CFRP Hybrid Cables", Arab J Sci Eng, vol. 45, p. 3569-3579, 2019, doi: 10.1007/s13369-019-04166-1.

[34] H. Dong, Y. Zhou y N. Zhuang, "Study on Corrosion Characteristics of Concrete-Filled CFRP-Steel Tube Piles under Hygrothermal Environment", $A d v$. Mater. Sci. Eng., no. 4849038, pp. 1-11, 2020, doi: 10.1155/2020/4849038.
[35] J. Zhou, X. Zhang, L. Zhang, F. Dong y E. Oh, "Static load tests of driven concrete piles under CFRP confinement", Geomech. Geoengin., pp. 1-14, 2019, doi: 10.1080/17486025.2019.1635713.

[36] A. Totonchi, A. Ansaripour y S. Shivaei, "Effect of Different Arrangements of CFRP Wraps on the Axial Stress-Strain Behaviour of Confined Concrete Cylinders: Experimental Study and Numerical Modelling", Iran J Sci Technol Trans Civ Eng, vol. 44, pp. 1087-1100, 2020, doi: 10.1007/s40996-019-00293-9.

[37] X. Lao, X. Han, J. Ji y B. Chen, "The compression behavior of CFRP-repaired damaged square RC columns", Constr Build Mater., vol. 223, pp. 1154-1166, 2019, doi: 10.1016/j.conbuildmat.2019.07.182.

[38] R. Z. Al-Rousan, "Impact of elevated temperature and anchored grooves on the shear behavior of reinforced concrete beams strengthened with CFRP composites", Case Stud. Constr. Mater., vol. 14, no. 487, pp. 1-21, 2021, doi: 10.1016/j.cscm.2021.e00487.

[39] F. Al-Mahmoud, A. Castel, T. Q. Minh y R. François, "Reinforced Concrete Beams Strengthened with NSM CFRP Rods in Shear", J. Sens., vol. 18, no. 10, pp. 15631574, 2015, doi: 10.1260/1369-4332.18.10.1563.

[40] F. Yu, S. Guo, S. Wang y Y. Fang, "Experimental study on high pre-cracked RC beams shear-strengthened with CFRP strips", Compos. Struct., vol. 225, no. 111163, pp. 1-14, 2019, doi: 10.1016/j.compstruct.2019.111163.

[41] A. Jawdhari, A. Peiris y I. Harik, "Experimental study on RC beams strengthened with CFRP rod panels", Eng. Struct., vol. 173, pp. 693-705, 2018, doi: 10.1016/j. engstruct.2018.06.105.

[42] H. Saleh, R. Kalfat, K. Abdouka y R. Al-Mahaidi, "Punching shear strengthening of RC slabs using L-CFRP laminates", Eng. Struct., vol. 194, pp. 279-289, 2019, doi: 10.1016/j.engstruct.2019.05.050.

[43] P. Preinstorfer, P. Huber, T. Huber, B. Kromoser y J. Kolleger, "Experimental investigation and analytical modelling of shear strength of thin walled textile-reinforced UHPC beams", Eng. Struct., vol. 231, no. 111735, pp. 1-11, 2021, doi: 10.1016/j. engstruct.2020.111735. 
[44] N. Moshiri, C. Czaderski, D. Mostofinejad y M. Motava1li, "Bond resistance of prestressed CFRP strips attached to concrete by using EBR and EBROG strengthening methods", Constr Build Mater., vol. 266, no. 121209, pp. 1-14, 2021, doi: 10.1016/j.conbuildmat.2020.121209.

[45] Y. M. Saeed, W. A. Aules, F. N. Rad y A. M. Raad, “Tensile behavior of FRP anchors made from CFRP ropes epoxy-bonded to uncracked concrete for flexural strengthening of RC columns", Case Stud. Constr. Mater., vol. 13, no. 435, pp. 1-19, 2020, doi: 10.1016/j.cscm.2020. e00435.

[46] M. Garg, C. S. Das y R. Gupta, "Use of silica particles to improve dispersion of $-\mathrm{COOH}$ CNTs/carbon fibers to produce HyFRCC", Constr Build Mater., vol. 250, no. 118777, pp. 1-15, 2020, doi: 10.1016/j. conbuildmat.2020.118777.

[47] R. Salih, F. Zhou, N. Abbas y A. K. Mastoi, "Experimental investigation of reinforced concrete beam with openings strengthened using FRP sheets under cyclic load", Materials, vol. 13, no. 3127, pp. 1-25, 2020, doi: 10.3390/ ma13143127.
[48] H. Elci, "Seismic strengthening of improperly repaired reinforced concrete columns using CFRP confinement", Structures, vol. 28, pp. 266-275, 2020, doi: 10.1016/j. istruc.2020.08.072.

[49] A. D. Mai, M. N. Sheikh, K. Yamakado y M. N. S. Hadi, "Nonuniform CFRP Wrapping to Prevent Sudden Failure of FRP Confined Square RC Columns", J. Compos. Constr., vol. 24, no. 6, pp. 1-12, 2020, doi: 10.1061/ (ASCE)CC.1943-5614.0001077.

[50] G. Habert, E. Denarié, A. Šajna y P. Rossi, "Lowering the global warming impact of bridge rehabilitations by using Ultra High Performance Fibre Reinforced Concretes", Cem Concr Compos, vol. 38, pp. 1-11, 2013, doi: 10.1016/j.cemconcomp.2012.11.008.

[51] A. O. Sojobi y K. M. Liew, "Flexural behaviour and efficiency of CFRP-laminate reinforced recycled concrete beams: Optimization using linear weighted sum method", Compos. Struct., vol. 260, no. 113259, pp. 1-11, 2020, doi: 10.1016/j.compstruct.2020.113259. 